\title{
Higher-order expansions for distributions of extremes from general error distribution
}

Pu Jia and Tingting $\mathrm{Li}^{*}$

\section{${ }^{*}$ Correspondence:}

tinalee@swu.edu.cn

School of Mathematics and

Statistics, Southwest University,

Chongqing, 400715, P.R. China

\section{空 Springer}

\begin{abstract}
In this short note, with optimal normalizing constants, the higher-order expansion for a distribution of normalized partial maximum from the general error distribution is derived, by which one deduces the associate convergence rate of the distribution of the extreme to the Gumbel extreme value distribution.
\end{abstract}

MSC: Primary 62E20; 60E05; secondary 60F15; 60G15

Keywords: expansion; extreme; general error distribution

\section{Introduction}

Let $\left\{X_{n}, n \geq 1\right\}$ be a sequence of independent and identically distributed random variables with marginal cumulative distribution function (cdf) $F_{v}$ following the general error distribution $\left(F_{v} \sim \operatorname{GED}(v)\right.$ for short), and let $M_{n}=\max _{1 \leq k \leq n} X_{k}$ denote the partial maximum of $\left\{X_{n}, n \geq 1\right\}$. The probability density function (pdf) of $\operatorname{GED}(v)$ is given by

$$
f_{v}(x)=\frac{v \exp \left(-(1 / 2)|x / \lambda|^{v}\right)}{\lambda 2^{1+1 / v} \Gamma(1 / v)}, \quad x \in \mathbb{R}
$$

where $v>0$ is the shape parameter, $\lambda=\left[2^{-2 / v} \Gamma(1 / v) / \Gamma(3 / v)\right]^{1 / 2}$ and $\Gamma(\cdot)$ denotes the Gamma function (Nelson [1]). Note that $\operatorname{GED}(2)$ reduces to the standard normal distribution.

Recently, several contributions investigated asymptotic behaviors of normalized maxima from the $\operatorname{GED}(v)$. It is well known that the limiting distribution of extremes from the $\operatorname{GED}(2)$, i.e., the normal distribution, is a Gumbel extreme value distribution, see Leadbetter et al. [2] and Resnick [3]. Peng et al. [4] established the Mills type ratio of GED $(v)$ and proved that there exist normalizing constants $a_{n}>0$ and $b_{n} \in \mathbb{R}$ such that

$$
\lim _{n \rightarrow \infty} \mathbb{P}\left(M_{n} \leq a_{n} x+b_{n}\right)=\lim _{n \rightarrow \infty} F_{v}^{n}\left(a_{n} x+b_{n}\right)=\Lambda(x)=\exp (-\exp (-x)), \quad x \in \mathbb{R},
$$

i.e., $F_{v}$ is in the domain of attraction of $\Lambda$, which we denote by $F_{v} \in D(\Lambda)$. For the uniform convergence rate of normalized maxima from the $\operatorname{GED}(v)$, Hall [5] established the optimal uniform convergence rate as $v=2$, i.e., the normal case; Peng et al. [6] extended the result to the case of $v>1$. Both studies show that the optimal convergence rate of extremes from the $\operatorname{GED}(v)$ is proportional to $1 / \log n$.

For more informative studies of extremes from the GED, Nair [7] considered higherorder expansions for distribution and moments of normalized maxima from the GED(2) 
under optimal normalizing constants. Let $\Phi(x)$ denote the distribution function of the standard normal distribution GED(2), Nair [7] proved that

$$
\tilde{b}_{n}^{2}\left[\tilde{b}_{n}^{2}\left(\Phi^{n}\left(\tilde{a}_{n} x+\tilde{b}_{n}\right)-\Lambda(x)\right)-\tilde{k}(x) \Lambda(x)\right] \rightarrow\left(\tilde{w}(x)+\frac{1}{2} \tilde{k}^{2}(x)\right) \Lambda(x)
$$

as $n \rightarrow \infty$, where the optimal normalizing constants $\tilde{a}_{n}$ and $\tilde{b}_{n}$ are given by

$$
1-\Phi\left(\tilde{b}_{n}\right)=n^{-1}, \quad \tilde{a}_{n}=\tilde{b}_{n}^{-1}
$$

Here, $\tilde{k}(x)$ and $\tilde{w}(x)$ are, respectively, of the following form:

$$
\tilde{k}(x)=2^{-1}\left(x^{2}+2 x\right) e^{-x}
$$

and

$$
\tilde{w}(x)=-8^{-1}\left(x^{4}+4 x^{3}+8 x^{2}+16 x\right) e^{-x} .
$$

In this short note, the aim is to establish a higher-order expansion for the distribution of normalized maxima from the $\operatorname{GED}(v)$ for $v>0$. For some recent related work on uniform convergence rates and higher-order expansions of extremes for given distributions, see Liao and Peng [8] for the log-normal distribution, and Liao et al. $[9,10]$ for skew distributions.

In order to derive the higher-order expansions of extremes from the $\operatorname{GED}(v)$, we cite some results from Peng et al. $[4,6]$. The following Mills ratio of the $\operatorname{GED}(v)$ is due to Peng et al. [4]:

$$
\frac{1-F_{v}(x)}{f_{v}(x)} \sim \frac{2 \lambda^{v}}{v} x^{1-v} \quad \text { as } x \rightarrow \infty,
$$

which deduces the following distributional tail representation of $\operatorname{GED}(v)$ :

$$
1-F_{\nu}(x)=c(x) \exp \left(-\int_{\lambda}^{x} \frac{g(t)}{f(t)} d t\right)
$$

for large $x>0$, where

$$
c(x) \rightarrow \frac{\exp (-1 / 2)}{2^{1 / v} \Gamma(1 / v)} \quad \text { as } x \rightarrow \infty
$$

and

$$
f(t)=2 v^{-1} \lambda^{v} t^{1-v}, \quad g(t)=1+2(v-1) v^{-1} \lambda^{v} t^{-v} .
$$

Noting that $f^{\prime}(t) \rightarrow 0$ and $g(t) \rightarrow 1$, we may choose normalizing constants $a_{n}$ and $b_{n}$ satisfying the following equations:

$$
1-F_{v}\left(b_{n}\right)=n^{-1}, \quad a_{n}=f\left(b_{n}\right) .
$$


Under these normalizing constants, we have

$$
\lim _{n \rightarrow \infty} F_{v}^{n}\left(a_{n} x+b_{n}\right)=\Lambda(x)
$$

This paper is organized as follows. Section 2 provides the main results. Some auxiliary results and the proofs of the main results are given in Section 3.

\section{Main result}

In this section, we provide asymptotic expansions of a distribution for the partial maximum of the GED with normalizing constants $a_{n}$ and $b_{n}$ given by (1.4).

Theorem 1 Let $F_{v}(x)$ denote the cdf of $\operatorname{GED}(v)$ with $v>0$. Then:

(i) For $v \neq 1$, with normalizing constants $a_{n}$ and $b_{n}$ given by (1.4), we have

$$
b_{n}^{v}\left[b_{n}^{v}\left(F_{v}^{n}\left(a_{n} x+b_{n}\right)-\Lambda(x)\right)-k_{v}(x) \Lambda(x)\right] \rightarrow\left(w_{v}(x)+\frac{k_{v}^{2}(x)}{2}\right) \Lambda(x)
$$

as $n \rightarrow \infty$, where $k_{v}(x)$ and $w_{v}(x)$ are, respectively, given by

$$
k_{v}(x)=\left(1-v^{-1}\right) \lambda^{v}\left(x^{2}+2 x\right) e^{-x}
$$

and

$$
w_{v}(x)=\left(v^{-1}-1\right) \lambda^{2 v}\left[4 x+2 x^{2}+\frac{2}{3}\left(2-v^{-1}\right) x^{3}+\frac{1}{2}\left(1-v^{-1}\right) x^{4}\right] e^{-x} .
$$

(ii) For $v=1$, with normalizing constants $a_{n}=2^{-1 / 2}$ and $b_{n}=2^{-1 / 2}(\log n-\log 2)$, we have

$$
e^{\sqrt{2} b_{n}}\left[e^{\sqrt{2} b_{n}}\left(F_{1}^{n}\left(a_{n} x+b_{n}\right)-\Lambda(x)\right)-k_{1}(x) \Lambda(x)\right] \rightarrow\left(w_{1}(x)+\frac{k_{1}^{2}(x)}{2}\right) \Lambda(x)
$$

as $n \rightarrow \infty$, where $k_{1}(x)$ and $w_{1}(x)$ are, respectively, given by

$$
k_{1}(x)=-\frac{1}{4} e^{-2 x}, \quad w_{1}(x)=-\frac{1}{12} e^{-3 x} .
$$

Remark 1 The main result coincides with (1.1) as the GED reduces to the standard normal distribution GED(2).

Remark 2 From (1.2) and (1.4), it is easy to check that $b_{n}^{v}=O(\log n)$. Hence, for $v \neq 1$, Theorem 1(i) shows that the convergence rate of $F_{v}^{n}\left(a_{n} x+b_{n}\right)$ to its ultimate extreme value distribution $\Lambda(x)$ is proportional to $1 / \log n$, while for the case of $v=1$, Theorem 1 (ii) shows that the convergence rate is proportional to $1 / n$.

\section{The proofs}

In order to prove the main results, we need some auxiliary lemmas. The first lemma deals with a decomposition of the distributional tail representation of $\operatorname{GED}(v)$. 
Lemma 1 Let $F_{v}(x)$ and $f_{v}(x)$, respectively, denote the cdf and $p d f$ of $\operatorname{GED}(v)$ with $v \neq 1$; for large $x>0$, we have

$$
\begin{aligned}
1-F_{v}(x)= & \frac{\exp (-1 / 2)}{2^{1 / v} \Gamma(1 / v)}\left[1+2\left(v^{-1}-1\right) \lambda^{v} x^{-v}\right. \\
& \left.+4\left(v^{-1}-1\right)\left(v^{-1}-2\right) \lambda^{2 v} x^{-2 v}+O\left(x^{-3 v}\right)\right] \exp \left(-\int_{\lambda}^{x} \frac{g(t)}{f(t)} d t\right)
\end{aligned}
$$

with $f(t)$ and $g(t)$ given by (1.3).

Proof Using integration by parts we have

$$
\begin{aligned}
1- & F_{v}(x) \\
= & \frac{v}{2^{1+1 / v} \Gamma(1 / v)} \int_{x / \lambda}^{\infty} \exp \left(-\frac{t^{v}}{2}\right) d t \\
= & f_{v}(x) \frac{2 \lambda^{v}}{v} x^{1-v}\left[1+2\left(v^{-1}-1\right) \lambda^{v} x^{-v}\right. \\
& \left.+4\left(v^{-1}-1\right)\left(v^{-1}-2\right) \lambda^{2 v} x^{-2 v}+8\left(v^{-1}-1\right)\left(v^{-1}-2\right)\left(v^{-1}-3\right) \lambda^{3 v} x^{-3 v}\right] \\
& +\frac{16 v}{2^{1+1 / v} \Gamma(1 / v)}\left(v^{-1}-1\right)\left(v^{-1}-2\right)\left(v^{-1}-3\right)\left(v^{-1}-4\right) \int_{x / \lambda}^{\infty} \exp \left(-\frac{t^{v}}{2}\right) t^{-4 v} d t .
\end{aligned}
$$

An application of L'Hospital's rule shows that

$$
\lim _{x \rightarrow \infty} \frac{\int_{x / \lambda}^{\infty} \exp \left(-\frac{t^{v}}{2}\right) t^{-4 v} d t}{\exp \left(-\frac{x^{\nu}}{2 \lambda^{\nu}}\right) x^{1-4 v}}=0 .
$$

Combining the latter with (1.2), (3.2), and (3.3), for large $x$ we have

$$
\begin{aligned}
1-F_{v}(x)= & f_{v}(x) \frac{2 \lambda^{v}}{v} x^{1-v}\left[1+2\left(v^{-1}-1\right) \lambda^{v} x^{-v}\right. \\
& \left.+4\left(v^{-1}-1\right)\left(v^{-1}-2\right) \lambda^{2 v} x^{-2 v}+O\left(x^{-3 v}\right)\right] \\
= & \frac{\exp (-1 / 2)}{2^{1 / v} \Gamma(1 / v)}\left[1+2\left(v^{-1}-1\right) \lambda^{v} x^{-v}\right. \\
& \left.+4\left(v^{-1}-1\right)\left(v^{-1}-2\right) \lambda^{2 v} x^{-2 v}+O\left(x^{-3 v}\right)\right] \exp \left(-\int_{\lambda}^{x} \frac{g(t)}{f(t)} d t\right),
\end{aligned}
$$

which is the desired result.

Lemma 2 Let $h_{v}\left(b_{n} ; x\right)=n \log F_{v}\left(a_{n} x+b_{n}\right)+e^{-x}$ with normalizing constants $a_{n}$ and $b_{n}$ given by (1.4), then for $v \neq 1$ we have

$$
\lim _{n \rightarrow \infty} b_{n}^{v}\left(b_{n}^{v} h_{v}\left(b_{n} ; x\right)-k_{v}(x)\right)=w_{v}(x),
$$

where $k_{v}(x)$ and $w_{v}(x)$ are given by Theorem 1. 
Proof It is well known that $n\left(1-F_{v}\left(a_{n} x+b_{n}\right)\right) \rightarrow e^{-x}$ as $n \rightarrow \infty$. By $1-F_{v}\left(b_{n}\right)=n^{-1}$, we know that $b_{n} \rightarrow \infty$ if and only if $n \rightarrow \infty$. The following fact holds by (1.2):

$$
\lim _{n \rightarrow \infty} \frac{1-F_{v}\left(a_{n} x+b_{n}\right)}{b_{n}^{-m v}}=0 \quad \text { for } m=1,2 .
$$

Let

$$
A_{v}(n, x)=\frac{1+\frac{2(1-v)}{v \lambda^{-v}} b_{n}^{-v}+\frac{4(1-v)(1-2 v)}{v^{2} \lambda^{-2 v}} b_{n}^{-2 v}+O\left(b_{n}^{-3 v}\right)}{1+\frac{2(1-v)}{v \lambda^{-v}}\left(a_{n} x+b_{n}\right)^{-v}+\frac{4(1-v)(1-2 v)}{v^{2} \lambda^{-2 v}}\left(a_{n} x+b_{n}\right)^{-2 v}+O\left(\left(a_{n} x+b_{n}\right)^{-3 v}\right)} .
$$

It is easy to check that $\lim _{n \rightarrow \infty} A_{v}(n, x)=1$ and

$$
A_{v}(n, x)-1=(1+o(1))\left[4 \frac{(1-v)}{v} \lambda^{2 v} b_{n}^{-2 v} x+16 \frac{(1-v)(1-2 v)}{v^{2}} \lambda^{3 v} b_{n}^{-3 v} x+O\left(b_{n}^{-3 v}\right)\right]
$$

Hence,

$$
\lim _{n \rightarrow \infty} \frac{A_{v}(n, x)-1}{b_{n}^{-v}}=0
$$

and

$$
\lim _{n \rightarrow \infty} \frac{A_{v}(n, x)-1}{b_{n}^{-2 v}}=4\left(v^{-1}-1\right) \lambda^{2 v} x .
$$

By (3.1) we have

$$
\begin{aligned}
& \frac{1-F_{v}\left(b_{n}\right)}{1-F_{v}\left(a_{n} x+b_{n}\right)} e^{-x} \\
& =A_{v}(n, x) \exp \left[\int_{0}^{x}\left(\frac{(v-1) a_{n}}{b_{n}+a_{n} t}+\frac{v a_{n}\left(b_{n}+a_{n} t\right)^{v-1}}{2 \lambda^{v}}-1\right) d t\right] \\
& =A_{v}(n, x)\left\{1+\int_{0}^{x}\left(\frac{(v-1) a_{n}}{b_{n}+a_{n} t}+\frac{v a_{n}\left(b_{n}+a_{n} t\right)^{v-1}}{2 \lambda^{v}}-1\right) d t\right. \\
& \left.+\frac{1}{2}\left[\int_{0}^{x}\left(\frac{(v-1) a_{n}}{b_{n}+a_{n} t}+\frac{v a_{n}\left(b_{n}+a_{n} t\right)^{v-1}}{2 \lambda^{v}}-1\right) d t\right]^{2}(1+o(1))\right\} \text {. }
\end{aligned}
$$

It follows from (3.5)-(3.8) that

$$
\begin{aligned}
& \lim _{n \rightarrow \infty} b_{n}^{v} h_{v}\left(b_{n} ; x\right) \\
& \quad=\lim _{n \rightarrow \infty} \frac{\log F_{v}\left(a_{n} x+b_{n}\right)+n^{-1} e^{-x}}{n^{-1} b_{n}^{-v}} \\
& \quad=\lim _{n \rightarrow \infty}\left[\frac{-\left(1-F_{v}\left(a_{n} x+b_{n}\right)\right)-\frac{1}{2}\left(1-F_{v}\left(a_{n} x+b_{n}\right)\right)^{2}(1+o(1))}{n^{-1} b_{n}^{-v}}+\frac{\left(1-F_{v}\left(b_{n}\right)\right) e^{-x}}{n^{-1} b_{n}^{-v}}\right] \\
& =\lim _{n \rightarrow \infty} \frac{\left(1-F_{v}\left(a_{n} x+b_{n}\right)\right)}{n^{-1}} \frac{\left(\frac{1-F_{v}\left(b_{n}\right)}{1-F_{v}\left(a_{n} x+b_{n}\right)} e^{-x}-1\right)}{b_{n}^{-v}} \\
& \quad=e^{-x} \lim _{n \rightarrow \infty}\left[\frac{A_{v}(n, x)\left[\int_{0}^{x}\left(\frac{(v-1) a_{n}}{b_{n}+a_{n} t}+\frac{v a_{n}\left(b_{n}+a_{n} t\right)^{v-1}}{2 \lambda^{v}}-1\right) d t\right](1+o(1))}{b_{n}^{-v}}+\frac{A_{v}(n, x)-1}{b_{n}^{-v}}\right]
\end{aligned}
$$




$$
\begin{aligned}
& =e^{-x} \lim _{n \rightarrow \infty} \int_{0}^{x} b_{n}^{v}\left(\frac{(v-1) a_{n}}{b_{n}+a_{n} t}+\frac{v a_{n}\left(b_{n}+a_{n} t\right)^{v-1}}{2 \lambda^{v}}-1\right) d t \\
& =\left(1-v^{-1}\right) \lambda^{v}\left(x^{2}+2 x\right) e^{-x}=k_{v}(x),
\end{aligned}
$$

where the last step is due to the dominated convergence theorem since

$$
\lim _{n \rightarrow \infty} b_{n}^{v}\left(\frac{v a_{n}\left(b_{n}+a_{n} t\right)^{v-1}}{2 \lambda^{v}}-1\right)=2\left(1-v^{-1}\right) \lambda^{v} t
$$

and

$$
\lim _{n \rightarrow \infty} \frac{(v-1) a_{n} b_{n}^{v}}{b_{n}+a_{n} t}=2\left(1-v^{-1}\right) \lambda^{\nu} .
$$

By arguments similar to (3.9), we have

$$
\begin{aligned}
& \lim _{n \rightarrow \infty} b_{n}^{v}\left(b_{n}^{v} h_{v}\left(b_{n} ; x\right)-k_{v}(x)\right) \\
& =\lim _{n \rightarrow \infty} \frac{\log F_{v}\left(a_{n} x+b_{n}\right)+n^{-1} e^{-x}-n^{-1} b_{n}^{-v} k_{v}(x)}{n^{-1} b_{n}^{-2 v}} \\
& =\lim _{n \rightarrow \infty}\left[\frac{-\left(1-F_{v}\left(a_{n} x+b_{n}\right)\right)-\frac{1}{2}\left(1-F_{v}\left(a_{n} x+b_{n}\right)\right)^{2}(1+o(1))}{n^{-1} b_{n}^{-2 v}}\right. \\
& \left.+\frac{n^{-1} e^{-x}-n^{-1} b_{n}^{-v} k_{v}(x)}{n^{-1} b_{n}^{-2 v}}\right] \\
& =\lim _{n \rightarrow \infty} \frac{-\left(1-F_{v}\left(a_{n} x+b_{n}\right)\right)+n^{-1} e^{-x}\left(1-k_{v}(x) e^{x} b_{n}^{-v}\right)}{n^{-1} b_{n}^{-2 v}} \\
& =\lim _{n \rightarrow \infty} \frac{1-F_{v}\left(a_{n} x+b_{n}\right)}{n^{-1}} \frac{\frac{1-F_{v}\left(b_{n}\right)}{1-F_{v}\left(a_{n} x+b_{n}\right)} e^{-x}\left(1-k_{v}(x) e^{x} b_{n}^{-v}\right)-1}{b_{n}^{-2 v}} \\
& =e^{-x} \lim _{n \rightarrow \infty}\left[A_{v}(n, x) b_{n}^{2 v}\left(\int_{0}^{x}\left(\frac{(v-1) a_{n}}{b_{n}+a_{n} t}+\frac{v a_{n}\left(b_{n}+a_{n} t\right)^{v-1}}{2 \lambda^{v}}-1\right) d t-k_{v}(x) e^{x} b_{n}^{-v}\right)\right. \\
& -k_{v}(x) e^{x} A_{v}(n, x) b_{n}^{v} \int_{0}^{x}\left(\frac{(v-1) a_{n}}{b_{n}+a_{n} t}+\frac{v a_{n}\left(b_{n}+a_{n} t\right)^{\nu-1}}{2 \lambda^{v}}-1\right) d t \\
& +\frac{1}{2}(1+o(1)) A_{v}(n, x) b_{n}^{2 v}\left(\int_{0}^{x}\left(\frac{(v-1) a_{n}}{b_{n}+a_{n} t}+\frac{v a_{n}\left(b_{n}+a_{n} t\right)^{v-1}}{2 \lambda^{v}}-1\right) d t\right)^{2} \\
& \left.+\frac{A_{v}(n, x)-1}{b_{n}^{-2 v}}\right] \\
& =\left(v^{-1}-1\right) \lambda^{2 v}\left[4 x+2 x^{2}+\frac{2}{3}\left(2-v^{-1}\right) x^{3}+\frac{1}{2}\left(1-v^{-1}\right) x^{4}\right] e^{-x} \\
& =w_{v}(x) .
\end{aligned}
$$

The proof is complete.

For $v=1$, noting that the $\operatorname{GED}(1)$ is the Laplace distribution with pdf given by

$$
f_{1}(x)=2^{-1 / 2} \exp \left(-2^{1 / 2}|x|\right), \quad x \in \mathbb{R},
$$


and the Laplace distributional tail can be written by

$$
1-F_{1}(x)=2^{-1 / 2} f_{1}(x)=2^{-1} \exp \left(-2^{1 / 2}\right) \exp \left(-\int_{1}^{x} \frac{1}{f(t)} d t\right), \quad x>0
$$

with $f(t)=2^{-1 / 2}$. For the Laplace distribution, we have the following result.

Lemma 3 For $v=1$, let $h_{1}\left(b_{n} ; x\right)=n \log F_{1}\left(a_{n} x+b_{n}\right)+e^{-x}$ with normalizing constants $a_{n}=$ $2^{-1 / 2}$ and $b_{n}=2^{-1 / 2}(\log n-\log 2)$. Then

$$
\lim _{n \rightarrow \infty} e^{\sqrt{2} b_{n}}\left(e^{\sqrt{2} b_{n}} h_{1}\left(b_{n} ; x\right)-k_{1}(x)\right)=w_{1}(x),
$$

where $k_{1}(x)$ and $w_{1}(x)$ are those given by Theorem 1.

Proof Noting that for $\operatorname{GED}(1)$, i.e., the Laplace distribution with pdf $f_{1}(x)=2^{-1 / 2} \times$ $\exp \left(-2^{1 / 2}|x|\right)$, we have

$$
\lim _{n \rightarrow \infty} F_{1}^{n}\left(a_{n} x+b_{n}\right)=\Lambda(x)
$$

with normalizing constants $a_{n}=2^{-1 / 2}$ and $b_{n}=2^{-1 / 2}(\log n-\log 2)$. So, by (1.4) and (3.13), we have

$$
\begin{aligned}
& \lim _{n \rightarrow \infty} e^{\sqrt{2} b_{n}} h_{1}\left(b_{n} ; x\right) \\
& \quad=\lim _{n \rightarrow \infty} \frac{\log F_{1}\left(a_{n} x+b_{n}\right)+n^{-1} e^{-x}}{n^{-1} e^{-\sqrt{2} b_{n}}} \\
& \quad=\lim _{n \rightarrow \infty}\left[\frac{-\left(1-F_{1}\left(a_{n} x+b_{n}\right)\right)-\frac{1}{2}\left(1-F_{1}\left(a_{n} x+b_{n}\right)\right)^{2}(1+o(1))}{n^{-1} e^{-\sqrt{2} b_{n}}}+\frac{\left(1-F_{1}\left(b_{n}\right)\right) e^{-x}}{n^{-1} e^{-\sqrt{2} b_{n}}}\right] \\
& =\lim _{n \rightarrow \infty} \frac{-\frac{1}{2}\left(1-F_{1}\left(a_{n} x+b_{n}\right)\right)^{2}(1+o(1))}{n^{-1} e^{-\sqrt{2} b_{n}}} \\
& =-\frac{1}{4} e^{-2 x}=k_{1}(x)
\end{aligned}
$$

and

$$
\begin{aligned}
\lim _{n \rightarrow \infty} e^{\sqrt{2} b_{n}}\left(e^{\sqrt{2} b_{n}} h_{1}\left(b_{n} ; x\right)-k_{1}(x)\right) \\
=\lim _{n \rightarrow \infty} \frac{\log F_{1}\left(a_{n} x+b_{n}\right)+n^{-1} e^{-x}}{n^{-1} e^{-2 \sqrt{2} b_{n}}-k_{1}(x) e^{\sqrt{2} b_{n}}} \\
=\lim _{n \rightarrow \infty}\left[\frac{-\left(1-F_{1}\left(a_{n} x+b_{n}\right)\right)-\frac{1}{2}\left(1-F_{1}\left(a_{n} x+b_{n}\right)\right)^{2}-\frac{1}{3}\left(1-F_{1}\left(a_{n} x+b_{n}\right)\right)^{3}(1+o(1))}{n^{-1} e^{-2 \sqrt{2} b_{n}}}\right. \\
\quad+\frac{\left(1-F_{1}\left(b_{n}\right)\right) e^{-x}}{\left.n^{-1} e^{-2 \sqrt{2} b_{n}}-k_{1}(x) e^{\sqrt{2} b_{n}}\right]} \\
=\lim _{n \rightarrow \infty} \frac{-\frac{1}{3}\left(1-F_{1}\left(a_{n} x+b_{n}\right)\right)^{3}(1+o(1))}{n^{-1} e^{-2 \sqrt{2} b_{n}}} \\
=-\frac{1}{12} e^{-3 x}=w_{1}(x) .
\end{aligned}
$$

The proof is complete. 
Proof of Theorem 1 By (3.9) and (3.15), we have

$$
h_{v}\left(b_{n} ; x\right) \rightarrow 0 \text { and }\left|\sum_{i=3}^{\infty} \frac{h_{v}^{i-3}\left(b_{n} ; x\right)}{i !}\right|<\exp \left(\left|h_{v}\left(b_{n} ; x\right)\right|\right) \rightarrow 1
$$

as $n \rightarrow \infty$. For the case of $v \neq 1$, by Lemma 2 and (3.17), we have

$$
\begin{aligned}
b_{n}^{v} & {\left[b_{n}^{v}\left(F_{v}^{n}\left(a_{n} x+b_{n}\right)-\Lambda(x)\right)-k_{v}(x) \Lambda(x)\right] } \\
& =b_{n}^{v}\left[b_{n}^{v}\left(\exp \left(h_{v}\left(b_{n} ; x\right)\right)-1\right)-k_{v}(x)\right] \Lambda(x) \\
& =\left[b_{n}^{v}\left(b_{n}^{v} h_{v}\left(b_{n} ; x\right)-k_{v}(x)\right)+b_{n}^{2 v} h_{v}^{2}\left(b_{n} ; x\right)\left(\frac{1}{2}+h_{v}\left(b_{n} ; x\right) \sum_{i=3}^{\infty} \frac{h_{v}^{i-3}\left(b_{n} ; x\right)}{i !}\right)\right] \Lambda(x) \\
& \rightarrow\left(w_{v}(x)+\frac{1}{2} k_{v}^{2}(x)\right) \Lambda(x)
\end{aligned}
$$

as $n \rightarrow \infty$. Similarly, by Lemma 3 and (3.17), we get

$$
e^{\sqrt{2} b_{n}}\left[e^{\sqrt{2} b_{n}}\left(F_{1}^{n}\left(a_{n} x+b_{n}\right)-\Lambda(x)\right)-k_{1}(x) \Lambda(x)\right] \rightarrow\left(w_{1}(x)+\frac{1}{2} k_{1}^{2}(x)\right) \Lambda(x)
$$

as $n \rightarrow \infty$.

The proof is complete.

\section{Competing interests}

The authors declare that they have no competing interests.

\section{Authors' contributions}

PJ obtained the theorem and completed the proof. TL corrected and improved the final version. Both authors read and approved the final manuscript.

\section{Acknowledgements}

This work was supported by the National Natural Science Foundation of China (11171275), the Natural Science Foundation Project of CQ (cstc2012jjA00029) and the Fundamental Research Funds for the Central Universities (XDJK2013C021, XDJK2014D020).

Received: 2 January 2014 Accepted: 14 May 2014 Published: 27 May 2014

\section{References}

1. Nelson, DB: Conditional heteroskedasticity in asset returns: a new approach. Econometrica 59, 347-370 (1991)

2. Leadbetter, MR, Lindgren, G, Rootzén, H: Extremes and Related Properties of Random Sequences and Processes. Springer, New York (1983)

3. Resnick, SI: Extreme Values, Regular Variation and Point Processes. Springer, New York (1987)

4. Peng, Z, Tong, B, Nadarajah, S: Tail behavior of the general error distribution. Econometrica 38, 1884-1892 (2009)

5. Hall, P: On the rate of convergence of normal extremes. J. Appl. Probab. 16, $433-439$ (1979)

6. Peng, Z, Nadarajah, S, Lin, F: Convergence rate of extremes for the general error distribution. J. Appl. Probab. 47, 668-679 (2010)

7. Nair, KA: Asymptotic distribution and moments of normal extremes. Stat. Probab. Lett. 9, 150-153 (1981)

8. Liao, X, Peng, Z: Convergence rate of limit distribution of maxima of lognormal samples. J. Math. Anal. Appl. 395, 643-653 (2012)

9. Liao, X, Peng, Z, Nadarajah, S: Tail properties and asymptotic expansions for the maximum of logarithmic skew-normal distribution. J. Appl. Probab. 50, 900-907 (2013)

10. Liao, $X$, Peng, Z, Nadarajah, S, Wang, $X$ : Rates of convergence of extremes from skew normal sample. Stat. Probab. Lett. 84, 40-47 (2014)

10.1186/1029-242X-2014-213

Cite this article as: Jia and Li: Higher-order expansions for distributions of extremes from general error distribution. Journal of Inequalities and Applications 2014, 2014:213 